\title{
Preferential localization of SSEA-4 in interfaces between blastomeres of mouse
}

\section{preimplantaion embryos}

\author{
Ban Sato ${ }^{1,5}$, Yohko U. Katagiri ${ }^{1,4}$, Kenji Miyado $^{2}$, Hidenori Akutsu ${ }^{2}$, Yoshitaka Miyagawa ${ }^{1}$, \\ Yasuomi Horiuchi ${ }^{1}$, Hideki Nakajima ${ }^{1}$, Hajime Okita ${ }^{1,4}$, Akihiro Umezawa ${ }^{2}$, Jun-ichi Hata ${ }^{1}$, \\ Junichiro Fujimoto ${ }^{3,4}$, Kiyotaka Toshimori ${ }^{5}$, Nobutaka Kiyokawa ${ }^{1,4}$ \\ ${ }^{1}$ Department of Developmental Biology, ${ }^{2}$ Department of Reproductive Biology, ${ }^{3}$ Vice General \\ Director, National Research Institute for Child Health and Development, 2-10-1 Okura,
}

Setagaya-ku, Tokyo 157-8535, Japan

${ }^{4}$ Core Research for Evolutional Science and Technology (CREST) of

Japan Science and Technology Corporation (JST)

${ }^{5}$ Department of Anatomy and Developmental Biology, Graduate School of Medicine, Chiba

University, Chiba 260-8670, Japan

\footnotetext{
" Corresponding author

Fax:+81-3-3417-2496.

e-mail address: kata@nch.go.jp (Y.U. Katagiri)

(C) 200. This manuscript version is made available under the Elsevier user license http://www.elsevier.com/open-access/userlicense $1.0 /$
} 


\section{Abstract}

The monoclonal antibody 6E2 raised against the embryonal carcinoma cell line

NCR-G3 had been shown to also react with human germ cells. Thin-layer chromatography

(TLC) immunostaining revealed that 6E2 specifically reacts with sialosylglobopentaosylceramide (sialylGb5), which carries an epitope of stage-specific embryonic antigen-4 (SSEA-4), known as an important cell surface marker of embryogenesis.

The immunostaining of mouse preimplantation embryos without fixation showed that the binding of $6 \mathrm{E} 2$ caused the clustering and consequent accumulation of sialylGb5 at the interface between blastomeres. These results suggest that SSEA-4 actively moves on the cell surface and readily accumulates between blastomeres after binding of $6 \mathrm{E} 2$.

Key words: sialylGb5; SSEA-4; embryonic stem cells; embryonal carcinoma cells; preimplantation embryo; immunostaining 


\section{Introduction}

Embryonal carcinoma (EC) cells isolated from teratocarcinomas have been shown to

possess pluri- or multi-potency in both mouse and human systems [1-3]. In mice, certain EC

cells as well as embryonic stem (ES) cells have been considered to be developmentally

equivalent to the inner cell mass of blastocysts [1]. These EC cells are useful for clarifying the

molecular characteristics of early embryonic cells and thus many efforts have been made to

establish EC cell lines and monoclonal antibodies (Mabs) that detect differentiation-related

molecules on EC cells. As a consequence, a number of stage-specific markers for

embryogenesis have been identified. Notably, it is important that this molecular information is

adapted to research on ES cells or mouse preimplantation embryos. Stage-specific embryonic

antigen (SSEA) -1, -3 and -4, as well as tumor rejection antigen (TRA) -1-60 and -1-81 [4],

have been used as stage-specific markers for embryogenesis, though their functional

significance in early development remains unclear. Interestingly, however, most of these

antigens are carbohydrates themselves or closely related to the carbohydrates carried on

glycosphingolipids (GSLs) and glycoproteins [5]. 
6E2 is a Mab established by immunizing with NCR-G3 cells, a previously

established multipotent human EC cell line capable of differentiating into trophoblastic cell

lineages other than somatic cells [3]. It has been revealed that $6 \mathrm{E} 2$ reacts with not only human

ECs, including NCR-G2 and 3 cells, but also other germ cell tumors, as well as normal human

germ cells such as spermatogonia and oocytes [6]. Although a previous study reported that

6E2 immunoprecipitates a cell surface protein having a molecular weight of approximately 80

$\mathrm{kDa}$ from ${ }^{125} \mathrm{I}$-labeled NCR-G3 cells, the specific antigen recognized by $6 \mathrm{E} 2$ still remains

unknown. To characterize the antigen-specificity of $6 \mathrm{E} 2$, we examined the reactivity of the

Mab with other cell lines using several distinct methods. In this paper, we present evidence

that $6 \mathrm{E} 2$ recognizes SSEA-4 carried by sialylGb5. Using 6E2, we determined the localization

of SSEA-4 in "living" mouse preimplantation embryos and observed its preferential localization

in interface between blastomeres. 


\section{Materials and methods}

\subsection{Cells, antibodies and animals}

The human renal carcinoma cell line ACHN was purchased from American Type

Culture Collection. The African green monkey kidney cell line Vero was a gift from Dr. T,

Takeda of Department of Infectious Diseases Research, National Children's Medical Research

Center, Tokyo, Japan. Cells were maintained in Dulbecco's modified Eagle's minimum

essential medium (DMEM) (Sigma Chem., St. Louis, MO) supplemented with 10\% fetal bovine

serum (FBS) (JRH Bioscience, Lenexa, KS). The human EC cell line NCR-G2 [3] was

cultured in a 1:1 mixture of DMEM and Ham's F12 medium (DMEM/F12) (Gibco, Grand

Island, NY) supplemented with 10\% FBS, non-essential amino acid solution (NEAA) (Gibco),

and Insulin-Transferin-Sodium Selenite media (Gibco). The cynomolgus monkey ES cell line

MCK-6 [7] were provided by Dr. Yasushi Kondo of Mitsubishi Tanabe Pharma Corporation.

ES cells were grown on mouse embryonic fibroblast feeder cells that were inactivated by

motomycin C in DMEM/F12 (Gibco) supplemented with $20 \%$ Knock-out serum replacement

(Invitrogen), penicillin/streptmycine (GIBCO), Glutamax (GIBCO), NEAA (GIBCO), sodium

pyruvate (GIBCO), 2-mercaptethanol (GIBCO), SR (GIBCO), hSPS (GIBCO), and bFGF 
(GIBCO). The cultures were performed at $37{ }^{\circ} \mathrm{C}$ in a $5 \% \mathrm{CO}_{2}$ incubator. The human venous blood from a healthy consenting volunteer was drawn in a heparin-coated syringe. The blood was spun at 3,000 rpm for $15 \mathrm{~min}$ and human red blood cells (hRBCs) were washed three times in phosphate buffered saline (PBS).

The conjugation of affinity-purified $6 \mathrm{E} 2$ (mouse $\left.\mathrm{IgG}_{3}, \kappa\right)$ [6] to the fluorescence reagent was performed with an Alexa Fluor ${ }^{\circledR} 488$ monoclonal antibody labeling kit (Molecular Probes, Eugene, OR.) according to the manufacturer's instructions. The anti-SSEA-4 Mabs used in this study were Raft.2 [8] and MC813-70 (R\&D Systems, Inc Minneapolis, MN). Alexa Fluor ${ }^{\circledR} 488$ goat anti-mouse IgG and Streptavidin Alexa Fluor ${ }^{\circledR} 568$ was purchased from Molecular probes.

$\mathrm{BDF}_{1}$ mouse were purchased from Clea Japan (Tokyo, Japan).

\subsection{TLC immunostaining of GSLS}

TLC immunostaining of GSLs from cultured cells and hRBCs was performed as previously described [9]. Reference GSLs were purchased from Matlayer, Inc. (Pleasant Gap, PA). SialylGb5 was purified from ACHN cells by preparative TLC. Purified GM1b was kindly provided by Dr. Nakamura of RIKEN, Saitama, Japan [10]. 


\section{3. Flow cytometry}

Cells were harvested and incubated with a primary antibody $(1 \mu \mathrm{g} / \mathrm{ml})$ for $1 \mathrm{~h}$ on ice,

followed by treatment with fluorescein isothiocyanate-conjugated goat anti-mouse immunoglobulins (Jackson Immunoresearch Laboratories, Inc. West Grove, PA) at a dilution of 1:50 and analyzed with an EPICS-XL flow cytometer (Beckman Coulter, Inc, Miami, FL).

\subsection{Dot blot analysis}

Purified sialylGb5 was serially diluted (0.1-60 ng) and vacuum blotted onto a PVDF membrane by using a 96-well format dot blot apparatus (Bio-Rad Laboratories, Richmond, CA).

The membrane was immunostained with the Mab 6E2 or MC813-70 $(0.5 \mu \mathrm{g} / \mathrm{ml})$ according to a previously described procedure [9]. The antibodies that bound to the membranes were visualized with ECL-plus Western Blotting Detection Reagents (GE Healthcare UK Ltd, Buckinghamshire, UK) and scanned with a LAS-1000 luminescent imaging analyzer (Fujifilm, Tokyo, Japan). Scanned images were analyzed using the software Image Gauge with which the LAS-1000 was equipped.

\subsection{Indirect immunostaining of cynomolgus monkey ES cells}

Cells were grown on a glass bottomed dish (IWAKI) for 3days and then these cells were 
fixed for 30min with 4\% paraformaldehyde in PBS and permeabilized with $0.2 \%$ TritonX-200

in PBS for 20min. Subsequently, the cells were washed three times with PBS for $5 \mathrm{~min}$ and blocked with 5\% normal goat serum in PBS for 30min. The fixed cells were incubated with anti-SSEA-4 antibodies or isotype-matched mouse IgG at a dilution of 1:300 for $2 \mathrm{~h}$, followed by incubation with Alexa Fluor ${ }^{\circledR}$ 488-conjugated goat anti-mouse IgG at a dilution of 1:300 for

30min. DAPI was used for counter staining of nuclei.

\subsection{Immunostaining of mouse preimplantation embryos}

Mouse preimplantation embryos were collected from superovulated mice.

Seven-week-old BDF1 female mouse were induced to superovulate with intraperitoneal injections of pregnant mare's serum gonadotropin (ASKA Pharmaceutical co., Ltd. Tokyo, Japan) (5 IU) and human chorionic gonadotropin (hCG) (ASKA Pharmaceutical co) (5 IU) $48 \mathrm{~h}$ apart and mated with individual BDF1 male mice after the hCG injection. The 2-cell, the 8-cell and the morula stage embryos were flushed out from oviducts at 36, 60, and $72 \mathrm{~h}$ after the hCG injection, respectively. Animals were treated according to the institutional animal care and use guidelines of National Research Institute for Child Health and Development.

Embryos immediately after being collected and those prefixed with $2 \%$ 
paraformaldehyde in HEPES buffered saline were incubated in $30 \mu 1$ drops of M16 medium containing $0.45 \mu \mathrm{g}$ of Alexa Fluor ${ }^{\circledR} 488$-conjugated $6 \mathrm{E} 2$ for $1 \mathrm{~h}$ or biotinylated MC813-70 for 1 h, treated with streptavidin Alexa Fluor ${ }^{\circledR} 568$ diluted 1:300, and then they were washed three times in $30 \mu \mathrm{l}$ drops of M16 medium. All staining steps were carried out at $37^{\circ} \mathrm{C}$ in a $\mathrm{CO}_{2}$ incubator for fresh embryos and at $4{ }^{\circ} \mathrm{C}$ for fixed embryos. The stained embryos were placed in drop of a M16 medium on glass-bottomed dishes (IWAKI, Tokyo, Japan), and were observed with a LSM510 Zeiss Confocal laser-scanning microscope (Carl Zeiss, Thornwood, NY) to obtain a field of view of the embryo only with a $40 \mathrm{x}$ objective lens. 


\section{Results and Discussion}

\subsection{E2 specifically binds to sialylGb5}

In order to examine whether the $80 \mathrm{kDa}$ membrane protein is recognized by $6 \mathrm{E} 2$, we performed a Western analysis of the cell lysates or their immunoprecipitates with 6E2. Since no significant signal was detected on the blot (data not shown), we examined TLC immunostaining of GSLs extracted from several 6E2-positive cell lines. ACHN cells showed the expression of comparable amounts of $\mathrm{Gb} 3, \mathrm{~Gb} 4, \mathrm{~Gb} 5$ and sialylGb5, whereas Vero cells and NCR-G2 cells expressed predominantly Gb3 (Fig. 1A). TLC immunostaining analysis revealed that $6 \mathrm{E} 2$ binds to a major slow-migrating GSL extracted from these three cell lines. The slow-migrating GSL was identified as sialylGb5, defined by the Mab Raft.2. We observed that $6 \mathrm{E} 2$ bound to sialylGb5 (LKE-antigen) of hRBCs [13] (Fig. 1B). Finally, we examined the reactivity of $6 \mathrm{E} 2$ with purified GSLs and found that the Mab reacts with purified sialylGb5, but not purified GM1b (Fig. 1C). These results indicate that 6E2 specifically binds to sialylGb5 and thus is an anti-SSEA-4 Mab. The $80 \mathrm{kDa}$ protein might be associated with sialylGb5 in NCR-G3 cells and thus co-immunoprecipitated by 6E2.

\subsection{Comparison of reactivity to sialyl Gb5 between $6 E 2$ and MC813-70}


MC813-70 established by immunizing with human EC cell lines has been most widely

used as an anti-SSEA-4 antibody (mouse $\left.\mathrm{IgG}_{3}, \kappa\right)$ [14]. Therefore we compared the reactivities of the Mabs 6E2 and MC813-70 by flow cytometry and dot-blot immunostaining. The fluorescence intensity obtained with 6E2 was stronger than that with MC813-70 in each cell line and hRBCs (Fig. 2A). A recent flow cytometric study showed that MC813-70 strongly stains hRBCs, but other anti-sialyGb5 Mabs do not [15]. However, our data indicate that $6 \mathrm{E} 2$ is more reactive than MC813-70. Next we compared the reactivity of the two Mabs with that of sialylGb5 by dot-blot immunostaining. Serially diluted sialylGb5 was dot-blotted onto a PVDF membrane, and the membrane was immunostained with the two Mabs. Both 6E2 and MC813-70 bound to more than $12 \mathrm{ng}$ of sialylGb5, but the signals induced by $6 \mathrm{E} 2$ were stronger than those induced by MC813-70 (Fig. 2B,C). Thus, in addition to the flow cytometric analysis, the reactivity of $6 \mathrm{E} 2$ with sialylGb5 was stronger than that of MC813-70 by dot-blot immunostaining.

\subsection{SSEA-4 Immunostaining of cynomolgus monkey ES cells}

To confirm whether Mab 6E2 reacts with SSEA-4 on monkey ES cells, we performed an indirect immunofluorescence staining of cynomolgus monkey ES cells with Mab 6E2 and 
MC813-70. Mab 6E2 reacted with monkey ES cells (Fig.3a) as well as MC-813-70 did

(Fig.3b). No difference in staining patterns of SSEA-4 between the two Mabs was observed.

Mab 6E2 certainly stained SSEA-4 on monkey ES cells.

\subsection{SSEA-4 immunostaining of "living" mouse preimplantation embryos without fixation}

During early embryogenesis in mice, SSEA-4 had been reported to be expressed in fertilized eggs with levels gradually increasing to the morula stage and then decreasing [5].

Thus we examined the expression and distribution of SSEA-4 in preimplantation mouse embryos by immunostaining with both 6E2 and MC813-70. Both Mabs evenly stained the whole surface membranes of fixed mouse embryos, and no difference in staining pattern between the two was observed (data not shown). In order to perform a time-course of SSEA-4 distribution in a viable state, we performed immunostaining of preimplantation embryos without fixation.

3D-images of the $6 \mathrm{E} 2$ staining pattern obtained by confocal laser scanning microscopic observation clearly showed the localization of SSEA-4 on mouse preimplantation embryos. Two-cell embryos showed patches of SSEA-4 over the whole surface membrane with some accumulation at the interface between blastomeres (Fig. 4a). In 8-cell embryos, the amount 
accumulated at interfaces was further increased, as if planer membranes separate each blastomere, and some large patches were internalized but others were left on the surface membranes (Fig. 4b). The amount of SSEA-4 concentrated at the interfaces in morula was not as significant as in 8-cell embryos but still clearly observed and some patches were internalized (Fig. 4c).

2D-images of embryos stained with 6E2 showed a marked accumulation of SSEA-4 at the interfaces between blastomeres (Fig. 4d-f). These results suggest that sialylGb5 actively moves during development and tends to accumulate where blastomeres come into contact with each other.

Interestingly, however, the staining pattern of SSEA-4 using MC813-70 was different from that using 6E2. MC813-70 evenly stained the surface and the interface between blastomeres of 2-cell embryos with patches (Fig. 4g), and the amount of SSEA-4 at interfaces was not significant (Fig. 4j). In 8-cell embryos, there were patches of SSEA-4 in the central area of the outer surface of each blastomere (Fig. 4h, indicated by arrows), but the 2D-image showed that clustering also occurred at surfaces facing blastocoels (Fig. 4k, indicated by arrowheads). In morula embryos, SSEA-4 was distributed on the surface in patches and was 
enriched at the boundaries between blastomeres on the outer surface (Fig. 4i, 1).

It remains unclear why the pattern of staining of mouse preimplantation embryos differs

between 6E2 and MC813-70. The composition of fatty acids in GSLs influences the binding

of antibodies $[16,17]$ or bacterial toxins [18]. SialylGb5 recognized by the two MAbs might

differ in composition of fatty acids, resulting in different immunostaining patterns. It was

reported that the clustering of sialylGb5 by a Mab induces the activation of

sialylGb5-associated kinases in raft microdomains of human mammary carcinoma cells, leading

to downstream signaling $[19,20]$. The clustering of sialyGb5 by $6 \mathrm{E} 2$ on preimplantation

mouse embryos may also induce the activation of some kinases, followed by downstream

signaling. Recently, Comisky et al. suggested that lipid rafts and their associated molecules

are spatiotemporally positioned to play a critical role in preimplantation developmental events

[21]. The patches or clusters of sialylGb5 shown in our study suggest the presence of lipid

rafts containing sialylGb5 on mouse embryos.

$6 \mathrm{E} 2$ has high affinity for sialylGb5 and can be effectively conjugated with fluorescence

reagents, leading to excellent staining of SSEA-4 in the surface membrane of "living" mouse

preimplantation embryos. 6E2 should be of use for research into lipid rafts in early 
development and of great advantage for the characterization of ES cells and EC cells. 


\section{Acknowledgments}

We thank S. Yamauchi for her excellent secretarial work. This work was supported in part by

grants from CREST of JST and the 3rd. term comprehensive 10-year-strategy for cancer control,

Research on Children and Families, Research on Human Genome Tailor made and Research on

Publicly Essential Drugs and Medical Devices, Health and Labour Sciences Research Grants

from the Ministry of Health, Labour and Welfare of Japan and a grant from The Japan Leukemia

Research Field. 


\section{References}

[1] B. Mintz, and K. Illmensee, Normal genetically mosaic mice produced from malignant teratocarcinoma cells. Proc Natl Acad Sci U S A 72 (1975) 3585-9.

[2] P.W. Andrews, I. Damjanov, D. Simon, G.S. Banting, C. Carlin, N.C. Dracopoli, and J. Fogh, Pluripotent embryonal carcinoma clones derived from the human teratocarcinoma cell line Tera-2. Differentiation in vivo and in vitro. Lab Invest 50 (1984) 147-62.

[3] J.F. Jun-ichi HATA, Eizaburo ISHII, Akihiro UMEZAWA, Yasuo KOKAI, Yoshie MATSUBAYASHI, Hiroshi ABE, Satoru KUSAKARI, Haruto KIKUCHI, Taketo YAMADA, and Tatsuya MARUYAMA, Differntiation of human germ cell tumor cells in vivo and in vitro. Acta Histochem Cytochem 25 (1992) 563-76.

[4] J.S. Draper, C. Pigott, J.A. Thomson, and P.W. Andrews, Surface antigens of human embryonic stem cells: changes upon differentiation in culture. J Anat 200 (2002) 249-58.

[5] T. Muramatsu, and H. Muramatsu, Carbohydrate antigens expressed on stem cells and early embryonic cells. Glycoconj J 21 (2004) 41-5.

[6] T. Nakano, A. Umezawa, H. Abe, N. Suzuki, T. Yamada, S. Nozawa, and J. Hata, A monoclonal antibody that specifically reacts with human embryonal carcinomas, spermatogonia and oocytes is able to induce human EC cell death. Differentiation 58 (1995) 233-40.

[7] M. Yamamoto, N. Tase, T. Okuno, Y. Kondo, S. Akiba, N. Shimozawa, and K. Terao, Monitoring of gene expression in differentiation of embryoid bodies from cynomolgus monkey embryonic stem cells in the presence of bisphenol A. J Toxicol Sci 32 (2007) 301-10.

[8] Y.U. Katagiri, K. Ohmi, C. Katagiri, T. Sekino, H. Nakajima, T. Ebata, N. Kiyokawa, and J. Fujimoto, Prominent immunogenicity of monosialosyl galactosylgloboside, carrying a stage-specific embryonic antigen-4 (SSEA-4) epitope in the ACHN human renal tubular cell line-a simple method for producing monoclonal antibodies against detergent-insoluble microdomains/raft. Glycoconj J 18 (2001) 347-53.

[9] Y.U. Katagiri, N. Kiyokawa, K. Nakamura, H. Takenouchi, T. Taguchi, H. Okita, A. Umezawa, and J. Fujimoto, Laminin binding protein, 34/67 laminin receptor, carries stage-specific embryonic antigen-4 epitope defined by monoclonal antibody Raft.2. Biochem Biophys Res Commun 332 (2005) 1004-11. 
[10] K. Nakamura, Y. Hashimoto, M. Suzuki, A. Suzuki, and T. Yamakawa, Characterization of GM1b in mouse spleen. J Biochem (Tokyo) 96 (1984) 949-57.

[11] The nomenclature of lipids (recommendations 1976). IUPAC-IUB Commission on Biochemical Nomenclature. J Lipid Res 19 (1978) 114-28.

[12] L. Svennerholm, Designation and schematic structure of gangliosides and allied glycosphingolipids. Prog Brain Res 101 (1994) XI-XIV.

[13] L.L. Cooling, and K. Kelly, Inverse expression of $\mathrm{P}(\mathrm{k})$ and Luke blood group antigens on human RBCs. Transfusion 41 (2001) 898-907.

[14] L.H. Shevinsky, B.B. Knowles, I. Damjanov, and D. Solter, Monoclonal antibody to murine embryos defines a stage-specific embryonic antigen expressed on mouse embryos and human teratocarcinoma cells. Cell 30 (1982) 697-705.

[15] L. Cooling, and D. Hwang, Monoclonal antibody B2, a marker of neuroendocrine sympathoadrenal precursors, recognizes the Luke (LKE) antigen. Transfusion 45 (2005) 709-16.

[16] K. Iwabuchi, and I. Nagaoka, Lactosylceramide-enriched glycosphingolipid signaling domain mediates superoxide generation from human neutrophils. Blood 100 (2002) 1454-64.

[17] K. Iwabuchi, Y. Zhang, K. Handa, D.A. Withers, P. Sinay, and S. Hakomori, Reconstitution of membranes simulating "glycosignaling domain" and their susceptibility to lyso-GM3. J Biol Chem 275 (2000) 15174-81.

[18] A. Kiarash, B. Boyd, and C.A. Lingwood, Glycosphingolipid receptor function is modified by fatty acid content. Verotoxin 1 and verotoxin $2 \mathrm{c}$ preferentially recognize different globotriaosyl ceramide fatty acid homologues. J Biol Chem 269 (1994) 11138-46.

[19] W.F. Steelant, Y. Kawakami, A. Ito, K. Handa, E.A. Bruyneel, M. Mareel, and S. Hakomori, Monosialyl-Gb5 organized with cSrc and FAK in GEM of human breast carcinoma MCF-7 cells defines their invasive properties. FEBS Lett 531 (2002) 93-8.

[20] S. Van Slambrouck, and W.F. Steelant, Clustering of monosialyl-Gb5 initiates downstream signalling events leading to invasion of MCF-7 breast cancer cells. Biochem J 401 (2007) 689-99.

[21] M. Comiskey, and C.M. Warner, Spatio-temporal localization of membrane lipid rafts in mouse oocytes and cleaving preimplantation embryos. Dev Biol 303 (2007) 727-39. 


\section{Legends}

Fig. 1. TLC immunostaining of GSLs prepared from cultured cells and hRBCs. GSLs extracted from cultured cells and hRBCs or purified GSLs were separated by TLC in a solvent system of chloroform/methanol/water containing $0.2 \% \mathrm{CaCl}_{2}(5: 4: 1, \mathrm{v} / \mathrm{v} / \mathrm{v})$. Plates were chemically stained with orcinol-sulfurnic acid or were immunostained with $6 \mathrm{E} 2$ and Raft.2.

Lane 1, ACHN; Lane 2, Vero; Lane 3, NCR-G2; Lane 4, hRBCs; Lane 5, GM1b; Lane 6, sialylGb5. Reference markers used were disialosyl gangliosides of GD3, GD1a, and GD1b (R1), monosialosyl gangliosides of GM3, GM2, and GM1 (R2), and neutral GSLs of GlcCer, LacCer, Gb3, and Gb4 (R3). The nomenclature for GSLs follows the recommendations [11] of the IUB, and the gangliosides nomenclature of Svennerholm [12] was used.

Fig. 2. Reactivity of 6E2 and MC813-70 with sialylGb5. A: Flow cytometric analysis of SSEA-4-positive cells with 6E2. NCR-G2 cells, ACHN cells, Vero cells, and hRBCs were stained with 6E2 (bold line) or MC813-70 (dotted line) and with a FITC-conjugated secondary antibody and analyzed by flow cytometry. B: An image of the dot-blot immunostaining of sialylGb5 obtained with a LAS-1000 luminescent imaging analyzer. C: Measurement of antibodies bound (6E2 : solid line, MC813-70 : broken line)

Fig. 3. Indirect immunostaining of cynomolgus monkey ES cell line CMK-6 with 6E2 
and MC813-70. The CMK-6 cells were stained with 6E2 (a), MC813-70 (b), or isotype-matched mouse $\operatorname{IgG}$ (c) and visualized with secondary antibodies (green), followed by counterstaining of nuclei with DAPI (blue). Scale bars $=200 \mu \mathrm{m}$.

Fig. 4. Immunostaining of SSEA-4 on mouse preimplantation embryos with 6E2 and MC813-70. The embryos at the 2-cell (a, d, g, j), the 8-cell (b, e, h, k), and the morula (c, f, i, 1) stages were stained with 6E2 (green) or MC813-70 (red). The panels designated 3D (a, b, c, g, h, i) are three-dimensional images reconstructed by stacking optical slice images using LSM software and the panels designated $2 \mathrm{D}(\mathrm{d}, \mathrm{e}, \mathrm{f}, \mathrm{j}, \mathrm{k}, \mathrm{l})$ are an overlay of a fluorescent image and a differential interference contrast micrograph. Scale bars $=20 \mu \mathrm{m}$. 
Fig. 1

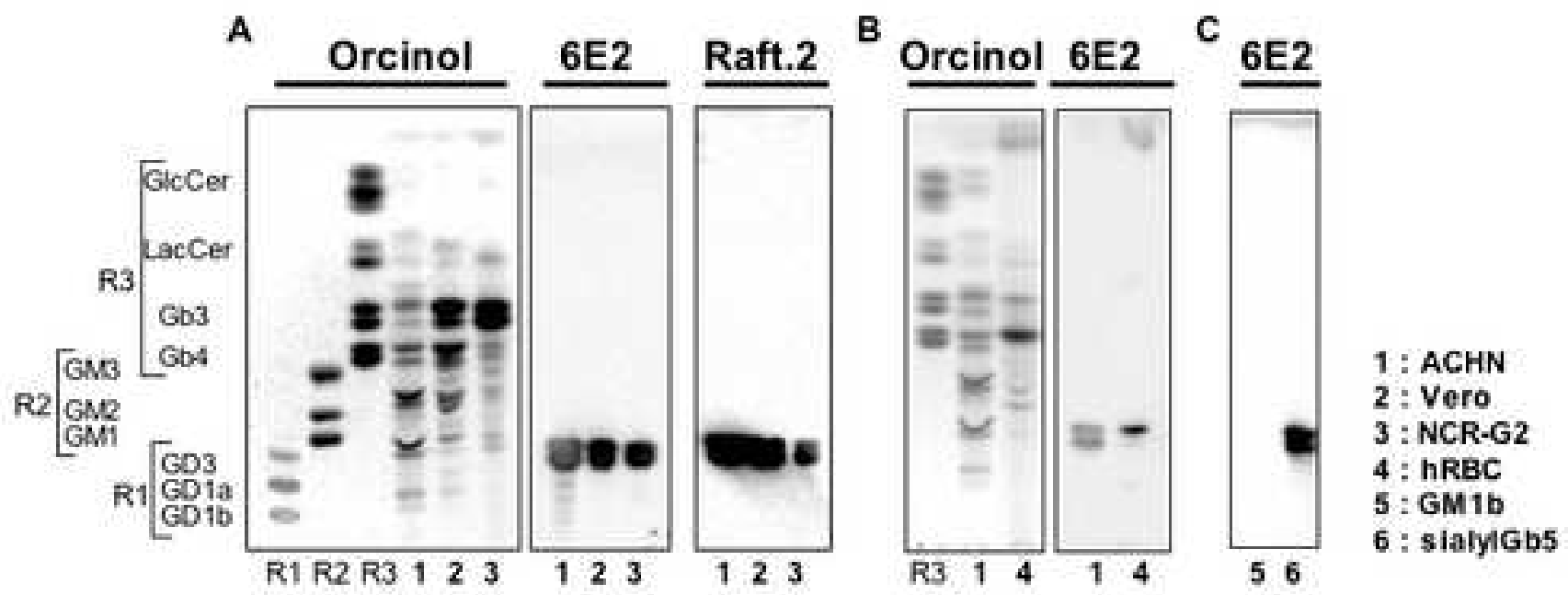


Fig.2

A

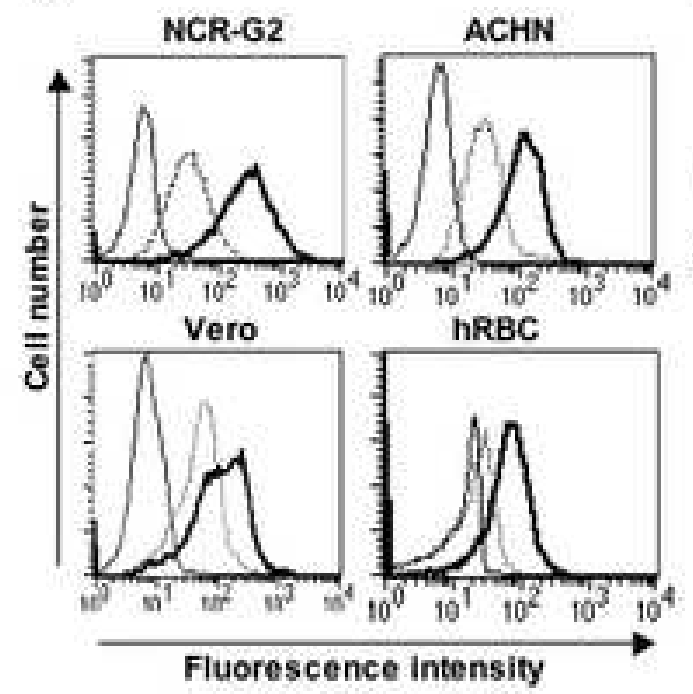

Negative control

MC-813-70

- 6E2
B

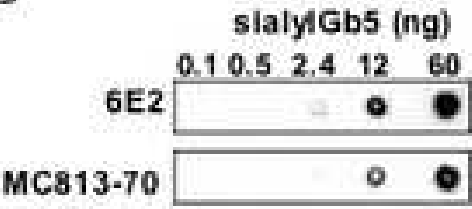

C

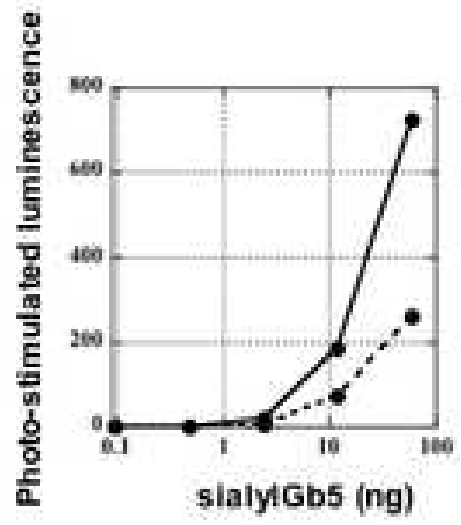


Fig. 3

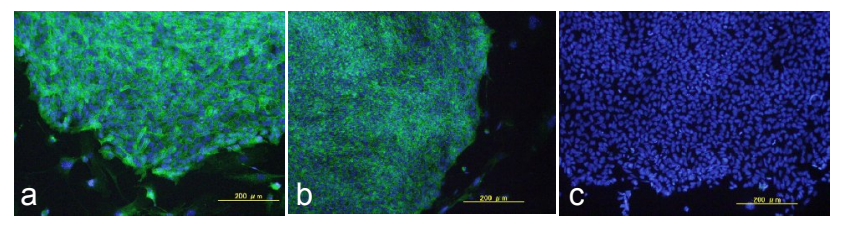


Fig. 4

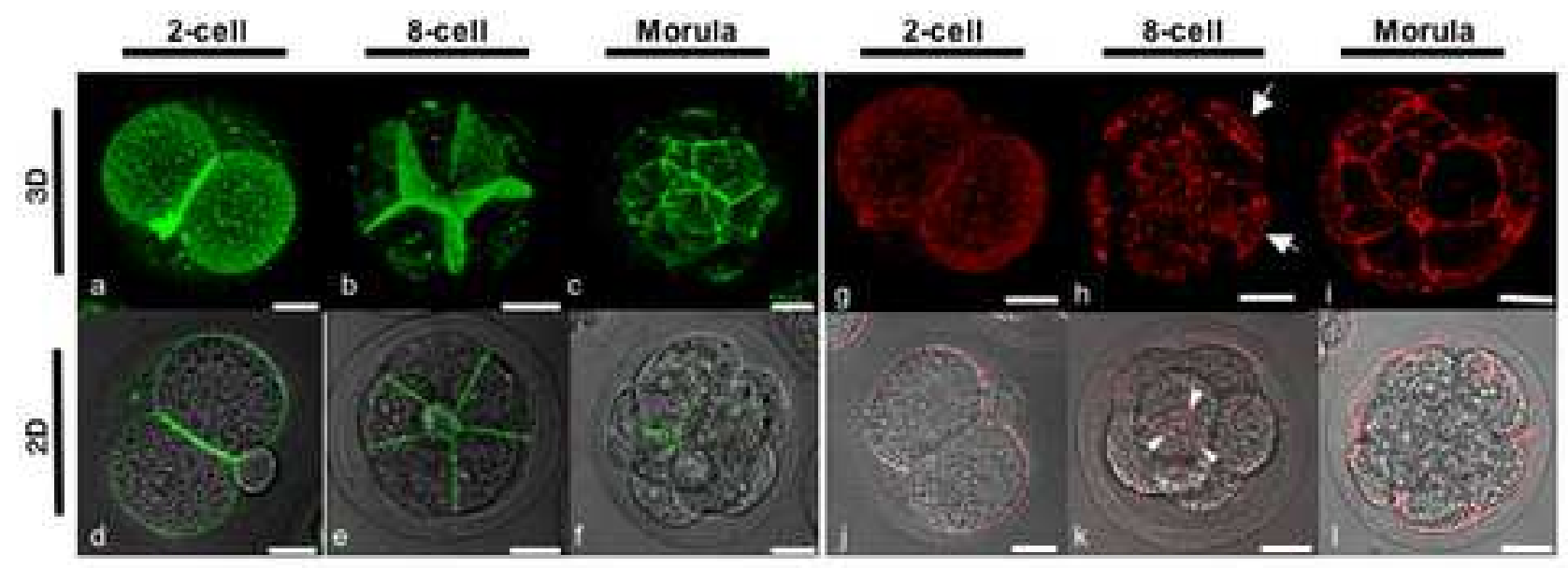

